weniger Politics scheint die Antwort auf die unbestreitbare Krise des Parteiensystems zu sein. In diesem Sinne wäre es lohnenswert, die Debatte fortzuführen.

Stephan Klecha

\section{Kruck, Andreas. Private Ratings, Public \\ Regulations. Credit Rating Agencies and Global Financial Governance.} Basingstoke. Palgrave/Macmillan 2011. 204 S. $100,00 \$$.

Das Wirken von Rating-Agenturen ist vor dem Hintergrund der jüngsten globalen Finanz- und Wirtschaftskrise Anstoß für Empörung und Kritik. Ihre politikwissenschaftliche Aufarbeitung steht allerdings erst am Anfang. Andreas Kruck leistet mit seinem bereits 2011 veröffentlichten Buch einen wichtigen Beitrag zur Klärung der Frage, warum Staaten überhaupt auf die Dienstleistungen von Rating-Agenturen zurückgreifen (9). Dabei vermeidet er es, in das Muster einseitiger Schuldzuweisungen zu verfallen. Verdeutlicht wird, dass die Stellung von RatingAgenturen durch politische Entscheidungen bewusst hervorgebracht wurde (11). Obwohl der Autor keine Diskussion der Strukturbedingungen des Finanzmarktkapitalismus vornimmt, erfüllt das Buch insgesamt seine im Titel anklingende Fragestellung klar und präzise. Private Rating-Agenturen sind Zulieferer einer Politik, die sich auf Ratings stützt und dadurch eine neue Form der Arbeitsteilung etabliert hat. Als Teil der Reihe Transformation des Staates, eines Sonderforschungsbereichs der Universitäten Bremen und Oldenburg, fügt sich die Arbeit in die Governance-Literatur und Vergleichende Politische Ökonomie ein (12f.). An- spruch und Durchführung machen das theoriegeleitete Buch eher für fortgeschrittene Studierende und Wissenschaftler interessant.

Die Arbeit gliedert sich in sechs Kapitel, wobei die Beschreibung des Gegenstands (Kapitel 2) allein ein gutes Drittel des knappen Umfangs ausmacht (1979) und sehr erschöpfend ausfällt. Es wird die historische Entwicklung von Rating-Agenturen, ihre innere Organisation, Einbettung in die Finanzmarktregulierung und der Bewertungsmechanismus dargestellt. Kruck hält fest, dass der Markt für Ratings oligopolistisch ist - gut 95 Prozent aller Bewertungen entfallen auf Analysen der Häuser "Moody's“, „Standard\&Poor's“ und „Fitch“ (30). Ihre Reichweite ist global, (41) ihre Güteprüfung zwingend. Bereits die Ankündigung der möglichen Abwertung eines Schuldtitels wirkt verhaltenssteuernd: „Even states might thus consider changing their behavior to suit the preferences of credit rating agencies [...]. “ (65). Ratings sind soziale Konstruktionen, die von den Finanzmarktakteuren geglaubt werden müssen (61). Diese Rolle verleiht politische Macht. Doch diese Rolle kommt nicht von ungefähr, sondern ist im Lauf der Zeit durch Aufgabendelegation auf die Agenturen übertragen worden (66ff.).

Der Autor will diese Delegation durch Theoriensynthese in den Kapitel drei bis fünf (80-155) aufarbeiten. Aus der Prinzipal-Agent-Theorie leitet er Verhaltenserwartungen und Kontrollprobleme bei der Vergabe öffentlicher Aufgaben an Private ab. Regierungen übertragen Aufgaben nur dann, wenn sie sich dazu nicht imstande sehen und der Gewinn an Handlungskapazität den erwarteten Verlust durch Delegation überwiegt (82-85). Dies erscheint widersprüchlich, doch die Einbindung 
von Rating-Agenturen in den Regulierungsprozess folgt einem systemischen Informationsbedarf in einer finanzialisierten Ökonomie (87). Staaten sollen Finanzmarktstabilität gewährleisten, Investitionssicherheit wird zum öffentlichen Gut. Und so werden Regierungen abhängig von den speziellen Angeboten der Agenturen. Ihre Wertschätzung privater Expertise folgt der Auffassung, dass der Markt für eine effektive Koordination sorgen kann. Der Bedarf an der Ressource "Rating“ nimmt in dem Maße zu, wie der Gesetzgeber mehr und mehr Wirtschaftszweige einem Monitoring durch externe Dritte unterwirft. Die Globalisierung des Anlagekapitals tritt als Verstärker hinzu (112-115). Dieses Muster ist nach Kruck zwischen den Volkswirtschaften unterschiedlich stark ausgeprägt, wie er mit Bezug auf den Varieties of Capitalism-Ansatz darstellt (117-123). In den USA wird stärker von Ratings Gebrauch gemacht als in Kontinentaleuropa, unterscheidet sich das Produktionsregime doch grundlegend.

Um diese Vermutung zu testen, führt Kruck im vierten Kapitel (131-144) eine vergleichende Analyse über Zeit und Raum durch. Der Bedeutungszuwachs von Ratings wird durch bekannte Fakten erklärt: Deregulierung der Finanzmärkte, Freigabe der Wechselkurse, Zunahme an Akteuren und komplexen Finanzprodukten, gestiegene Liquidität mit kurzfristiger Anlageperspektive und daraus folgende Kapitalvolatilität, die mit Verweis auf John Ruggie als „disembedded liberalism“ (132) gedeutet werden. Dies ist aus der Diskussion um Bedeutung und Entwicklung des „Neoliberalismus“ vertraut. Was fehlt, ist eine Diskussion der Einkommens- und Vermögensentwicklung in den entwickelten OECD-Staaten; auch die Durchsetzung von Austeritätsprogrammen und die Nivellierung gesellschaftlicher Machtressourcen, wie sie die Staatstätigkeitsforschung hervorhebt, fehlt in dieser eher institutionentheoretischen Analyse und muss an anderer Stelle, etwa in jüngeren Beiträgen zu dieser Zeitschrift, nachgeschlagen werden. Interessanter fällt der regionale Vergleich aus (139-144), da Kruck hier die Möglichkeiten und Grenzen der Abweichung von privaten Ratings darstellt. In den USA ist dies nahezu unmöglich, da der Markt an Finanzprodukten wesentlich größer, unübersichtlich strukturiert und höchst risikoanfällig ist (140), was der Handel mit Derivaten und die daraus folgende Subprime-Krise im Jahr 2007 aufzeigt. In der EU galt zumindest bis zur Implementation des Basel-II-Standards eine moderate Abhängigkeit von externen Ratings, da andere Informationsangebote zur Verfügung standen und Unternehmen bei der Kreditbeschaffung gar keiner Ratingpflicht unterlagen (141f.). Diese Differenz wird im fünften Kapitel (145-154) ausführlicher dargestellt. Hier rekonstruiert Kruck die Beweggründe, die bis 2004 zur Revision des alten Accounting-Standards führten. Bemerkenswert ist die veränderte Risikowahrnehmung der Politik seit Ende der 1990er Jahre, die sich eindeutig für eine Übertragung der Verantwortung auf Externe aussprach (147). Das markiert einen wesentlichen Unterschied zu anderen Governance-Formen wie Public-Private-Partnerships. Auch Deutschland war nicht grundsätzlich gegen eine solche Kompetenzverlagerung, wollte aber die privilegierte Stellung der Banken nicht aufgeben (152). Wettbewerbsnachteile für kleine und mittlere Unternehmen wegen höherer 
Eigenkapitalquoten wurden befürchtet (153). Schließlich wurden Zugeständnisse ausgehandelt, aber die Pflicht zur Bewertung der Risiken dennoch eingeführt und die Stellung der Agenturen bestätigt.

Die folgende Finanzkrise thematisiert der Autor erst am Schluss. Dabei werden nur die Maßnahmen angeführt, die US-Börsenaufsicht und EU-Kommission bis 2009 vor allem in technischer Hinsicht vorgenommen haben (158). Eine Kritik der Ratingpraxis bleibt Desiderat. Was Kruck aufgrund des Erscheinungsdatums nicht analysieren konnte und daher annonciert (163ff.), das sind die Reaktionen der EUROStaaten auf die Folgen der anschließenden Bankenrettungsprogramme. Das Narrativ der „Staatsschuldenkrise“ hat sich durchgesetzt. In diesem Kontext ist die Bedeutung von Rating-Agenturen eher noch gewachsen. Gerade die Finanzkrise des griechischen Staates zeigt die Reaktionshandlungen der Politik vor dem Hintergrund drohender Bonitätsabwertungen durch die großen Agenturen. Das ist ein Umstand, der die grundlegende Erklärungslogik des Buches stützt und die Lektüre dennoch empfehlenswert macht.

Alexander Leipold

\section{INTERNATIONALE BEZIEHUNGEN}

Mello, Patrick. Democratic Participation in Armed Conflict: Military Involvement in Kosovo, Afghanistan, and Iraq. Basingstoke. Palgrave (Palgrave Studies in International Relations) 2014. 280 Seiten. 87,99€.

Die Militärinterventionen seit dem Ende des Ost-West-Konflikts sind treffend als „wars of choice“ beschrieben worden. Unbeschadet bündnispolitischer und -solidarischer Erwägungen lassen sie im Gegensatz zur Territorialund Bündnisverteidigung jedem einzelnen Staat erhebliche Spielräume bei der Entscheidung, selbst einen militärischen Beitrag zu leisten - oder eben davon abzusehen. Die Forschung zum so genannten „Demokratischen Frieden“ hat uns dabei gelehrt, mit Unterschieden zwischen Demokratien und Autokratien zu rechnen, hat aber auch den Blick auf die auffälligen Differenzen zwischen liberalen Demokratien verstellt. Das ist schon deshalb bedauerlich, weil die Unterschiede zwischen Demokratien für Theorien des Konfliktverhaltens ebenso bedeutsam sind wie ihre Gemeinsamkeiten im Vergleich zu Autokratien.

Die bisherige Forschung spiegelt unterschiedliche methodische Vorlieben diesseits und jenseits des Atlantiks wider: In den USA arbeitende Kollegen haben das Konfliktverhalten von Demokratien bevorzugt quantitativ und am liebsten gleich über die letzten fünfzig oder hundert Jahre untersucht. In Europa beheimatete Autoren hingegen neigen zu Zweifeln, ob auf diese Weise generalisierbare Befunde gewonnen werden können, und legen daher lieber qualitative Studien zum Konfliktverhalten einzelner oder weniger Demokratien in einzelnen oder wenigen Konflikten vor.

Patrick Mellos Studie zeichnet sich dadurch aus, die Vorzüge quantitativer und qualitativer Zugänge miteinander $\mathrm{zu}$ verbinden. In drei Fallstudien zu den Militärinterventionen im Kosovo 1999, in Afghanistan 2001 und im Irak 2003 werden die Beteiligungen von über zwanzig liberalen Demokratien verglichen. Im Gegensatz zu einigen quantitativen Studien werden „wars of 